\title{
Oxidative Stress and Inflammatory Markers PTX3, CypA, and HB-EGF: How Are They Linked in Patients With STEMI?
}

Angiology

2020, Vol. 7I(8) 7I3-720

(C) The Author(s) 2020

Article reuse guidelines:

sagepub.com/journals-permissions DOI: I0.II77/000331972092I724

journals.sagepub.com/home/ang

@SAGE

\author{
Vesna Vuković Dejanović, MSc' ${ }^{\circledR}$, Jelena Kotur Stevuljević, PhD², \\ Aleksandra Vukašinović, PhD $^{2}$, Milica Miljković, PhD $^{2}$, \\ Srdjan Kafedzic, MSc ${ }^{3,4}$, Marija Zdravković, $\mathrm{PhD}^{4,5}$, Ivan Ilić, $\mathrm{MSc}^{3,4}$, \\ Saša Hinić, $\mathrm{MSc}^{5}$, Milivoje Cerović, $\mathrm{MSc}^{3}$, Milica Stefanović, $\mathbf{P h D}^{3}$, \\ Vesna Spasojević-Kalimanovska, PhD $^{2}$, Lidija Memon, PhD $^{5}$, \\ Aleksandar N. Nešković, PhD ${ }^{3,4}$, and Nataša Bogavac-Stanojević, PhD ${ }^{2}$
}

\begin{abstract}
We investigated circulating levels of inflammatory biomarkers pentraxin-3 (PTX3), cyclophilin A (CypA), and heparin-binding epidermal growth factor-like growth factor (HB-EGF); oxidative stress; and antioxidant status markers in the patients with ST-segment elevation acute myocardial infarction (STEMI) to better understand a relationship between inflammation and oxidative stress. We examined the impact of oxidative stress on high values of inflammatory parameters. The study included 87 patients with STEMI and 193 controls. We observed a positive correlation between PTX3 and HB-EGF $(\rho=0.24, P=.027$ ), CyPA, and sulfhydryl $(\mathrm{SH})$ groups $(\rho=0.25, P=.026)$, and a negative correlation between $\mathrm{PTX} 3$ and SH groups $(\rho=-0.35, P=$ $.00 \mathrm{I}$ ) in patients with STEMI. To better understand the effect of the examined parameters on the occurrence of high concentrations of inflammatory parameters, we grouped them using principal component analysis. This analysis identified the 4 most contributing factors. Optimal cutoff values for discrimination of patients with STEMI from controls were calculated for PTX3 and HB-EGF. An independent predictor for PTX3 above the cutoff value was a "metabolic-oxidative stress factor" comprised of glucose and oxidative stress marker prooxidant-antioxidant balance (odds ratio $=4.449, P=.030$ ). The results show that higher PTX3 values will occur in patients having STEMI with greater metabolic and oxidative stress status values.
\end{abstract}

\section{Keywords}

pentraxin-3, cyclophilin A, heparin-binding EGF-like growth factor, ST-segment elevation acute myocardial infarction, oxidative stress

\section{Introduction}

Imbalance of pro- and antioxidants in favor of prooxidants, manifested as oxidative stress along with low-grade chronic inflammation, plays a role in the development of atherosclerosis, atherosclerosis plaque instability, and consequently acute myocardial infarction (AMI). ${ }^{1}$ Additionally, reactive oxygen species (ROS) are generated in AMI, especially after reperfusion. ${ }^{2}$ Oxidative stress and chronic inflammation are closely related ${ }^{3}$ and better understanding of their relationship may improve knowledge regarding the progression of cardiovascular disease (CVD).

This study analyzed 3 emerging biomarkers associated with inflammation and oxidative stress: cyclophilin A (СypA), pentraxin-3 (PTX3), and heparin-binding -like growth factor (HB-EGF). These parameters are involved in different stages of AMI development; however, the mechanisms of their activation are not mutually exclusive.

Cyclophilin A promotes cardiovascular (CV) inflammation, myocardial ischemia-reperfusion injury and responses to oxidative stress. Cyclophilin A is released by vascular smooth muscle cells (VSMCs) as a response to ROS, and vice versa, it stimulates VSMC proliferation and migration of inflammatory cells. ${ }^{4}$ Reduction in CyPA concentration decreases the expression of inflammatory cytokines such as

\footnotetext{
'Department of Cardiology Rehabilitation, Institute for Rehabilitation, Belgrade, Serbia

${ }^{2}$ Department of Medical Biochemistry, Faculty of Pharmacy, University of Belgrade, Belgrade, Serbia

${ }^{3}$ Clinical Hospital Center Zemun, Belgrade, Serbia

${ }^{4}$ Faculty of Medicine, University of Belgrade, Belgrade, Serbia

${ }^{5}$ Clinical Hospital Center Bezanijska Kosa, Belgrade, Serbia
}

Corresponding Author:

Vesna Vuković Dejanović, Department of Cardiology Rehabilitation, BiochemicalHaematological Laboratory, Institute for Rehabilitation, Sokobanjska 17, II000 Belgrade, Serbia.

Email: vdvesna@gmail.com 
interleukin 6 and the accumulation of inflammatory cells in the vascular wall and consequently reduces infarct size in animal model of ischemia-reperfusion injury. ${ }^{5}$ On the other hand, an increase in CyPA levels may indicate elevated oxidative stress and proinflammatory status in patients with AMI.

Proinflammatory stimuli activate inflammatory cells which further triggers the nuclear factor $\kappa \mathrm{B}(\mathrm{NF}-\kappa \mathrm{B})$ pathway leading to the production of tumor necrosis factor $\alpha$ (TNF- $\alpha$ ), interleukin $1 \beta$ (IL-1 $\beta$ ), and consequently to oxidative stress development. ${ }^{6}$ Tumor necrosis factor $\alpha$ and IL-1 $\beta$ directly or indirectly activate the transcription of HB-EGF and PTX3. ${ }^{7,8}$ Currently, there is no consensus about the roles of HB-EGF and PTX3 in AMI. There is evidence that they have a positive and detrimental role in this process. Some studies pointed that a high plasma concentration of HB-EGF originating from VSMC is associated with the presence of a more stable plaque phenotype, giving it an important role in protecting against CVD. ${ }^{9}$ The other studies reported that the treatment of arterial stenosis with angioplasty with or without stent implantation leads to restenosis, and HB-EGF is thought to lead to VSMC proliferation and intimal hyperplasia after a balloon injury on a restenosis animal model. ${ }^{10}$ Pentraxin-3 decreases proinflammatory and prothrombotic effect in AMI by reducing neutrophil infiltration at the site of myocardial damage and by binding to activated platelets. ${ }^{11}$ Some studies have shown significant relationship between PTX3 and high-risk plaque morphology, such as plaque rupture with possible embolization. ${ }^{12}$

High concentrations of all 3 biomarkers were recorded after AMI, and in most studies, they were associated with a worse AMI outcome. ${ }^{5,13,14}$ However, modulation of high concentrations of these parameters and overall impact of these changes is still unknown. Understanding which ROS are related to high CyPA, PTX3, and HB-EGF values in real-world circumstances would be very beneficial for future examinations of clinical utility values of these biomarkers.

Most published works evaluated the relationship between these parameters using single or multiple association models based on researchers' subjective opinion or evidence-based judgment. Simultaneously analyzing numerous variables is always associated with a risk of model overfitting and erroneous conclusions. By reducing the number of original variables, fewer relationships between variables can be established and probability for model overfitting is lower. Principal component analysis (PCA) methodology combines initial predictors in smaller number of sets and it allows better understanding how each variable is associated and related with another variable. ${ }^{15}$

Our aim was to assess whether PTX3, HB-EGF, and CyPA concentrations are higher in the patients with ST-segment elevation AMI (STEMI) compared with controls. Another goal was to determine which ROS and antioxidant markers are changed in AMI. We also aimed to investigate potential of oxidative stress and antioxidant markers to predict the occurrence of the high values of investigated inflammatory parameters in patients with STEMI.

\section{Material and Methods}

\section{Participants}

The study group included 87 patients with STEMI (60 males and 27 females; mean age $60.2 \pm 12.3$ ) admitted with the diagnoses of STEMI during the period from May 2016 until July 2017. STEMI was diagnosed in patients with characteristic clinical symptoms, persistent electrocardiographic ST-segment elevation in $\geq 2$ contiguous precordial or adjacent limb leads, followed by a rise in cardiac markers. Myocardial necrosis was diagnosed by troponin I (TnI) determination, and myocardial infarction was angiographically confirmed. Most of the patients with STEMI were on antihypertensive drug therapy ( $\beta$-blockers and angiotensin-converting enzyme inhibitors), combined with regular aspirin but without history of previous coronary artery disease. In all, 52 patients were treated at the Clinical Hospital Center "Bezanijska Kosa" and 35 patients at the Clinical Hospital Center Zemun. The control group consisted of 193 healthy volunteers (73 males and 120 females, mean age $56.1 \pm 10.2$ years), employees of the Faculty of Pharmacy in Belgrade, and persons who checked-in for regular medical examination in a general hospital "Medigroup" in Belgrade. Selection criteria for the control group were age $>50$ years, no history of a recent clinical infections in the renal system and hepatic or malignant disease, surgery, or major trauma $\geq 1$ month prior to enrollment. The control group underwent cardiology examination (electrocardiogram, stress test for coronary ischemia, and transthoracic echocardiography) that excluded CVD. Included study groups were matched by age.

Information about health conditions on studied patients was obtained from their medical records. Healthy individuals in the control group completed a questionnaire about their current medications use and smoking habits. Body mass index (BMI) for each individual was calculated using body weight $(\mathrm{kg})$ divided by the square of the height $(\mathrm{m})$. All patients and volunteers gave their informed consent prior to enrollment in the study. The study was planned according to the Declaration of Helsinki guidelines. ${ }^{16}$ The study protocol was approved by the ethical committees of Zemun and "Bezanijska Kosa" clinical centers as well as the Faculty of Pharmacy and the General Hospital "Medigroup" following local biomedical research regulations.

\section{Measurements}

Blood samples were collected from patients immediately after their admission to the emergency units, before carrying out primary percutaneous coronary intervention. An average of 1 hour and 30 minutes elapsed from the onset of chest pain to blood sampling. Blood samples were obtained from controls in a regular medical checkup appointment, after overnight fasting. Peripheral venous blood samples were drawn into collection tubes containing serum-separator gel for serum samples and 
EDTA as anticoagulant for plasma samples. Serum and plasma samples were separated from blood cells immediately after centrifugation, and oxidative stress status parameters and antioxidant status markers were determined in the serum samples of patients and controls. The EDTA plasma was stored at $-80^{\circ} \mathrm{C}$ until glucose, lipid status parameters, PTX3, CyPA, and HB-EGF were determined.

Troponin I was determined in serum samples of patients right after admission at emergency units and several times during hospitalization using electrochemiluminescence method (high sensitive TnI using a VIDAS analyzer, BioMérieux, Lyon, France).

Most of the biochemical and oxidative stress markers were measured using an ILAB 600 analyzer (Instrumentation Laboratory, Milan, Italy). Glucose, total cholesterol (TC), and triglycerides (TG) were assessed using a routine enzymatic method; high-density lipoprotein cholesterol (HDL-C) was quantified using a direct enzymatic method.

As samples from patients with STEMI were collected in nonfasting conditions, instead of calculating low-density cholesterol values, we calculated non-HDL-C values according to recommendations of European Society of Cardiology. ${ }^{17}$ NonHDL-C values were obtained by subtracting HDL-C from TC values. For the determination of advanced oxidation protein products (AOPP), the Witko-Sarsat et al ${ }^{18}$ method is used. The total content of SH groups was determined by Ellman method, ${ }^{19}$ which is based on the reaction of $2,2^{\prime}$-dinitro-5, $5^{\prime}$ dithionitrobenzoic acid with aliphatic thiol compounds in an alkaline environment.

The determination of total oxidant status (TOS) assumed that total oxidants in the sample oxidize the ferro-ortho-dianiside complex in a ferric ion, which then builds a colored complex with xylenol-orange that is measured at $560 \mathrm{~nm}$ wavelength. ${ }^{20}$ Prooxidant-antioxidant balance (PAB) was determined using $3,3^{\prime}, 5,5^{\prime}$-tetramethylbenzidine and its cation as a redox indicator by the method proposed by Alamdaria et al. ${ }^{21}$ The concentration of the present antioxidants in the form of total antioxidant status (TAS) was determined by Erel method. ${ }^{22}$ The determination of the activity of superoxide dismutase (SOD) was performed according to the method of Misra and Fridovich. ${ }^{23}$ Pentraxin3, HB-EGF, and CyPA were measured by enzyme-linked immunosorbent assay (Human Pentraxin3 DuoSet ELISA R\&D Systems, Minneapolis; Human HB-EGF DuoSet ELISA R\&D Systems, Minneapolis; Human cyclophilin A (PPIA/ CYPA) ELISA kit, CUSABIO Biotech Co, Ltd. (Serbia)).

\section{Statistical Analysis}

The Kolmogorov-Smirnov test was used to assess normality distribution of data. Data are shown as mean \pm standard deviation for continuous variables with normal distribution, while categorical variables are presented as relative and absolute frequencies. As distributions for all parameters except for age, BMI, and TC were skewed, data were presented as median and interquartile range. Normally distributed continuous variables were compared using Student $t$ test, and other continuous variables were tested by the Mann-Whitney test. Due to the small number of patients in the STEMI group and in order to maintain the statistical power of the study, the Mann-Whitney exact test was performed according to recommendations. ${ }^{24}$ Categorical variables were analyzed by Chi-square tests using contingency tables. Correlation between tested parameters was investigated by Spearman correlation analysis. We conducted a receiver operating characteristic (ROC) curve analysis in order to define high values of PTX3, CypA, and HB-EGF. We calculated the area under ROC (AUC) and evaluated the accuracy of examined parameters to distinguish patients with STEMI from apparently healthy people. For inflammatory parameters with statistically significant accuracy, we calculated optimal cutoff value based on maximal Youden index. For calculated cutoff value, the difference between parameter sensitivity (Se) or true positive rate and 1-specificity ( $\mathrm{Sp}$ ) or false positive rate was maximal. ${ }^{25}$ The following step in data analysis involved PCA with varimax-normalized rotation to reduce the number of examined variables to a smaller number of factors. The processed data included normally distributed variables and variables with skewed distribution after log transformation. An extracted factor was determined based on eigenvalues $>1$. Variables with factor loadings $\geq 0.5$ were used for the interpretation of factors. Scores were calculated for factors with eigenvalues $>1$ and those factors were included as independent variables in further analysis. Binary logistic regression was performed for the determination of significant predictors of high inflammatory parameter values.

The analyses were performed with PASW Statistic v. 25 (Chicago, Illinois) software. A 2-tailed $P<.05$ was considered significant.

\section{Results}

\section{Comparison of Tested Parameters Between STEMI and Control Groups}

Table 1 shows the demographic characteristics and levels of analyzed markers in control and STEMI groups. Except for non-HDL-C, CypA, and TOS, all other examined laboratory markers were significantly different between patients and controls. Systolic and diastolic blood pressure, glucose, TG, PTX3, and HB-EGF concentrations were higher, while TC and HDL-C concentrations were lower in STEMI group than in the controls. Regarding the parameters for oxidative stress, the STEMI group had a higher value of PAB, while concentrations of AOPP were lower in patients with STEMI than in controls. The antioxidant enzyme SOD was significantly increased, while SH groups content and TAS were decreased in patients with STEMI compared with controls. In addition, the prevalence of males and smokers was higher in patients with STEMI compared with controls.

\section{Correlation Between Inflammmatory and Oxidative Stress Parameters}

We also investigated the correlation between examined inflammatory markers (PTX3, CyPA, and HB-EGF), oxidative stress 
Table I. Demographic Characteristics and Circulating Levels of Examined Parameters in Patients With STEMI and Control Group. ${ }^{\mathrm{a}}$

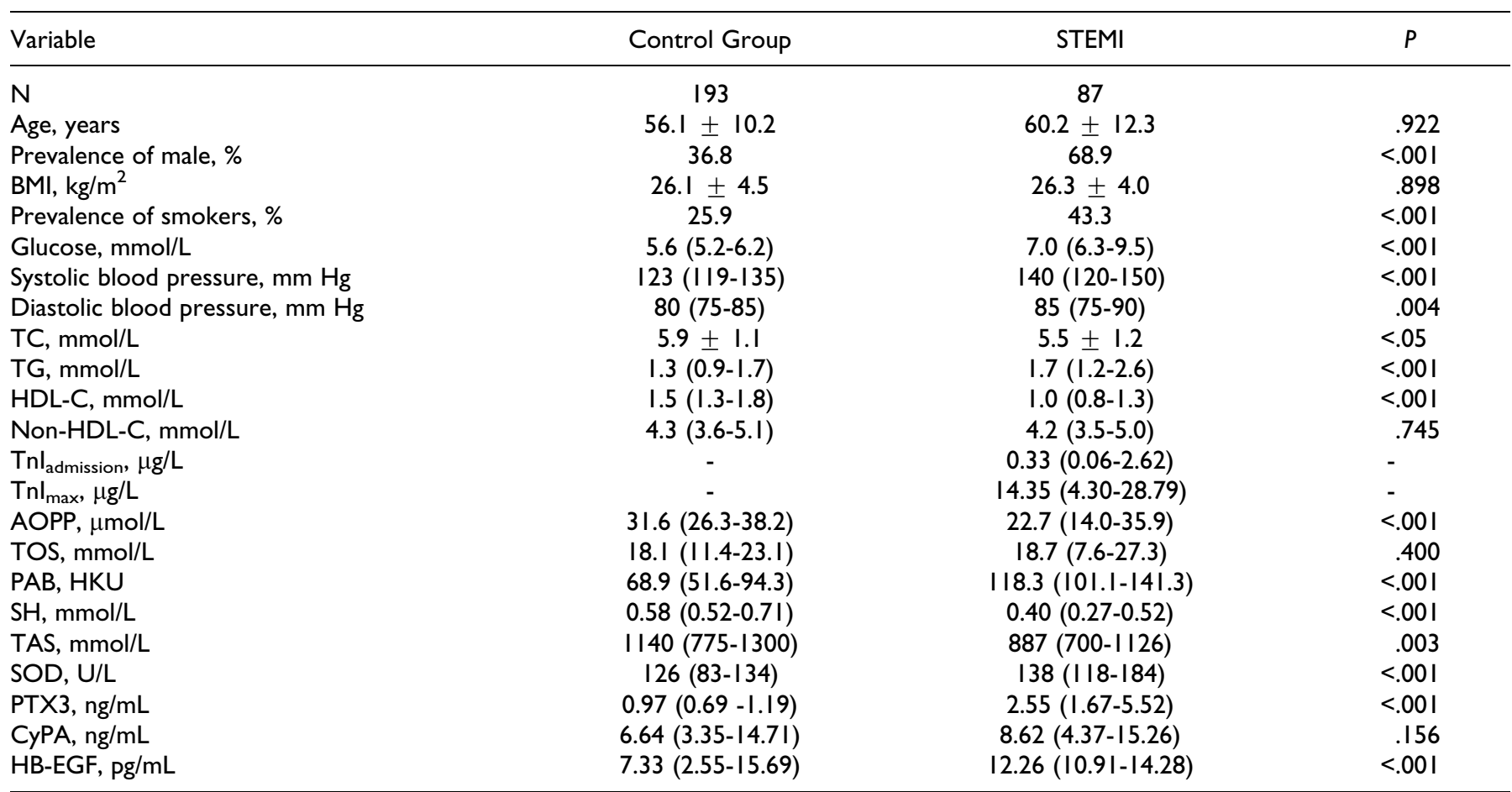

Abbreviations: AOPP, advanced oxidation protein products; BMI, body mass index; CyPA, cyclophilin A; HB-EGF, heparin-binding -like growth factor; HDL-C, HDL-cholesterol; non-HDL-C, non-HDL-cholesterol; PAB, prooxidant antioxidant balance; PTX3, pentraxin-3; SD, standard deviation; SH, sulphydryl groups; SOD, superoxide dismutase; STEMI, ST-segment elevation myocardial infarction; TAS, total antioxidant status; TC, total cholesterol; TG, triglycerides; Tnl admission, troponin I on admission at emergency unit; $\mathrm{TnI}_{\max }$, maximum troponin I value; TOS, total oxidant status.

${ }^{a}$ Continuous variables were compared by Student $t$ test or by Mann-Whitney exact test, and categorical variables by Chi-square test. Normally distributed continuous variables are presented as mean $\pm S D$, and categorical variables are presented as absolute and relative frequencies; variables with skewed distribution were presented as median and interquartile range.

Table 2. Optimal Cutoff Values and AUC of Inflammatory Parameters for Distinguishing Patients With STEMI From Healthy Individuals.

\begin{tabular}{lccccrrr}
\hline Variable & Cutoff Values & AUC & $95 \% \mathrm{Cl}$ & Sensitivity & Specificity & SD & $P$ \\
\hline PTX3, ng/mL & 1.61 & 0.944 & $0.90-0.99$ & 0.772 & 1.000 & 0.024 & $<.001$ \\
CyPA, ng/mL & 3.57 & 0.552 & $0.39-0.71$ & 0.845 & 0.733 & 0.082 & .492 \\
HB-EGF, pg/mL & 10.41 & 0.814 & $0.64-0.99$ & 0.810 & 0.778 & 0.088 & $<.001$ \\
\hline
\end{tabular}

Abbreviations: AUC, area under curve; Cl, confidence interval; CyPA, cyclophilin A; HB-EGF, heparin-binding epidermal growth factor-like growth factor; PTX3, pentraxin-3; SD, standard deviation; STEMI, ST-segment elevation myocardial infarction.

parameters (AOPP, TOS, PAB), markers of antioxidant status (SH, TAS, SOD), and glucose in patients with STEMI. Spearman correlation analysis showed positive correlation for CyPA and negative correlation of PTX3 with SH groups $(r=0.248$, $P=.026$ and $r=-0.349, P=.001$, respectively). Heparinbinding epidermal growth factor-like growth factor correlated positively with PTX3 $(r=0.240, P=.027)$. Similar findings for these parameters were obtained when processing the results of tests for the control group. The SH-group content correlated negatively with PTX3 $(r=-0.191, P=.023)$.

\section{Defining the High Values of Inflammatory Parameters}

Optimal cutoff values were calculated by ROC curve analyses: $1.61 \mathrm{ng} / \mathrm{mL}(\mathrm{Se}=0.772$ and $\mathrm{Sp}=1.000)$ and $10.41 \mathrm{pg} / \mathrm{mL}(\mathrm{Se}$ $=0.810$ and $\mathrm{Sp}=0.778)$ for PTX3 and HB-EGF, respectively.
The calculated AUC showed that both parameters had a good capability to discriminate patients with STEMI from controls (Table 2). Calculated AUC for PTX3 was $0.944(P<.001)$ and for HB-EGF was $0.814(P<.001)$. Cyclophilin A was not able to discriminate patients with STEMI from apparently healthy people (AUC $=0.552$ [95\% confidence interval: 0.392-0.712], $P=.492)$. High CyPA values were not defined due to low discriminative ability.

\section{Combining Initial Variables in Smaller Number of Factors}

Principal component analysis was applied to oxidative stress and antioxidant parameters and glucose. Four extracted factors explained $70 \%$ of the variance in all examined parameters. The first factor explained $21 \%$ of the total variance and it was associated with positive loading of AOPP and SH groups. 
Table 3. Extracted Factors by PCA.

\begin{tabular}{lcc}
\hline Factors & $\begin{array}{c}\text { Included Variables } \\
\text { With Loadings }\end{array}$ & $\begin{array}{c}\text { Factor } \\
\text { Variability }\end{array}$ \\
\hline Protein-related factor & AOPP $(0.812)$ & $21 \%$ \\
Metabolic-oxidative stress factor & $\begin{array}{c}\text { SH groups }(0.86 \mathrm{I}) \\
\text { Glucose }(0.775)\end{array}$ & $17 \%$ \\
Prooxidant-antioxidant balance factor & PAB $(0.733)$ & $17 \%$ \\
Age-related antioxidant factor & TOS $(0.818)$ & $10.718)$ \\
& Age $(0.767)$ & $15 \%$ \\
& TAS $(0.757)$ & \\
\hline
\end{tabular}

Abbreviations: AOPP, advanced oxidation protein products; $\mathrm{PAB}$, prooxidant antioxidant balance; $\mathrm{PCA}$, principal component analysis; $\mathrm{SH}$, sulphydryl groups; SOD, superoxide dismutase; TAS, total antioxidant status; TOS, total oxidant status.

We interpreted this behavior as a "protein-related factor." The second factor was characterized by glucose and PAB with positive loadings, and we name it as "metabolic-oxidative stress factor." It accounted for $17 \%$ of the total variance, similar to the third factor in the list. The third factor was comprised of TOS with positive loadings and SOD with negative loadings. The fourth factor explained $15 \%$ of the total variance, and it demonstrated positive loadings of age and TAS. The third and fourth factors were named as "prooxidant-antioxidant balance factor" and "age-related antioxidant factor," respectively. The results of PCA analysis are presented in Table 3.

\section{The Predictive Abilities of Determined Factors on High Values of PTX3 and HB-EGF}

To determine which factor is associated with high PTX3 and HB-EGF values in patients with STEMI, we applied a binary logistic regression. Results showed that significant predictors of PTX3 values $>1.61 \mathrm{ng} / \mathrm{mL}$ were metabolic-oxidative stress parameters, while for HB-EGF, there was no association between high values $(>10.41 \mathrm{pg} / \mathrm{mL})$ and examined factors. In addition, we examined the predictive abilities of HB-EGF and CypA on high PTX3 values and predictive abilities of PTX 3 and CypA on high HB-EGF values. According to the results presented in Table 4, the predictor of high PTX3 value was CypA and the predictor of high HB-EGF values was PTX3.

When significant predictors from an univariate logistic regression were included in multivariate regression, the independent predictor of PTX3 high values was the factor which was defined by metabolic-oxidative stress parameters (odds ratio $=4.449, P=.030$ ). Increased metabolic-oxidative stress parameter values were associated with a 4.5 greater probability for high PTX3 values.

\section{Discussion}

The aim of this study was to determine whether the values of PTX3, HB-EGF, and CyPA change due to AMI, and to determine possible correlation between examined inflammatory
Table 4. Univariate Logistic Regression Analysis of Predictors of High PTX3 and HB-EGF Values.

\begin{tabular}{|c|c|c|}
\hline Predictors & $\begin{array}{c}\text { High Values of PTX3 } \\
\text { OR }(95 \% \mathrm{Cl})\end{array}$ & $\begin{array}{c}\text { High Values of HB-EGF } \\
\text { OR }(95 \% \mathrm{Cl})\end{array}$ \\
\hline $\begin{array}{l}\text { Protein-related } \\
\text { factor }\end{array}$ & $0.918(0.497-1.693)$ & $\mathrm{I} .72 \mathrm{I}(0.78 \mathrm{I}-3.792)$ \\
\hline $\begin{array}{l}\text { Metabolic-oxidative } \\
\text { stress factor }\end{array}$ & $4.449(1.34|-| 4.760)^{a}$ & $1.288(0.546-3.038)$ \\
\hline $\begin{array}{l}\text { Prooxidant- } \\
\text { antioxidant } \\
\text { balance factor }\end{array}$ & $0.918(0.47 \mid-1.789)$ & $\mathrm{I} .06 \mathrm{I}(0.465-2.42 \mathrm{I})$ \\
\hline $\begin{array}{l}\text { Age-related } \\
\text { antioxidant } \\
\text { factor }\end{array}$ & $0.579(0.283-1.184)$ & $0.950(0.392-2.306)$ \\
\hline СурА & $0.932(0.878-0.990)^{a}$ & $1.023(0.933-1.121)$ \\
\hline HB-EGF & $0.994(0.895-1.105)$ & - \\
\hline PTX3 & - & $1.427(1.010-2.014)^{a}$ \\
\hline
\end{tabular}

Abbreviations: $\mathrm{Cl}$, confidence interval; CyPA, cyclophilin A; HB-EGF, heparinbinding epidermal growth factor-like growth factor; OR, odds ratio; PTX3, pentraxin-3.

${ }^{\text {a }}$ Significance at $P<.05$.

markers and oxidative stress. To investigate whether changes in PTX3, HB-EGF, and CyPA concentrations in patients with STEMI are a consequence of imbalance between oxidant and antioxidant stress parameters, these parameters were grouped into several different data sets (clusters) and their significance as predictors for high values of inflammatory related parameters was analyzed.

In patients with STEMI, we found significantly elevated values of PTX3 and HB-EGF compared with controls (Table 1). Our results are in accordance with other studies ${ }^{26-29}$ which also found increased values of PTX3 in patients with AMI. Only a few studies examined HB-EGF in patients with AMI and they indicated HB-EGF upregulation after AMI. ${ }^{9,30}$ Several studies determined a high expression of CypA in coronary atherosclerotic plaques in patients with $\mathrm{AMI}^{5,31}$; however, we did not observe a significant difference in circulating levels of CyPA between control and STEMI groups in the present study. Although most of oxidative stress parameters were altered, the effects of the oxidative stress on CyPA found in other studies were not confirmed in ours. It remains to be clarified whether this is due to the insufficient Se of CyPA to oxidative stress or whether this relationship becomes more pronounced after a certain time (because studies may vary in sampling time).

Numerous studies observed an increase in ROS relative to antioxidants in patients with AMI. ${ }^{32,33}$ However, there is no general consensus regarding the change of specific ROS and antioxidants markers in AMI. In the present project, patients with STEMI had elevated PAB values and decreased AOPP values compared with the control group. Considering that $\mathrm{PAB}$ represents a measure of concurrently prooxidant activity and antioxidant capacity of plasma, the increased values in our patients with STEMI indicate increased prooxidant activity and this is consistent with another study. ${ }^{34}$ As other researchers pointed out, we suggest that $\mathrm{PAB}$ could be used as a parameter 
for evaluation of oxidative stress status in patients with STEMI. ${ }^{35}$ On the other hand, AOPP are created as a result of protein damage by free radicals, especially albumin, during oxidative stress. Albumin is a negative acute phase reactant and the most abundant reduced thiol in human plasma containing a sulfhydryl group. ${ }^{36}$ With the decrease in albumin content during the acute phase of STEMI, it can be expected that AOPP production will be reduced, and its reduction in patients with STEMI is a possible indicator of severity in the acute phase.

Our results revealed a significant change in antioxidant marker levels within the STEMI group compared with healthy individuals. Total SH-group concentration was significantly lower in the STEMI group. The SH groups are typically derived from different amino acids (eg, cysteine and methionine) and their derivatives, such as homocysteine and glutathione. In oxidative stress conditions, SH groups could be oxidized and thus removed from the antioxidant components pool. The results from this study are in accordance with the results of others, ${ }^{37,38}$ indicating that oxidative stress reduces SH-groups content. This deterioration of antioxidant capacity has also been confirmed in our study by TAS decreasing in patients with STEMI. Toxic effects of ROS cause damage to all cellular components, including DNA, lipids, and proteins. As oxidative stress is increased in patients with STEMI, it is expected that the antioxidant system will be compromised. Measurement of TAS in plasma presents a method for studying antioxidants compiled from all blood biomolecules with reductive capacity. Our findings from this study are in a good accordance with the results of LoPresti et al. ${ }^{33}$ Total antioxidant capacity presents an action of several different substances: glutathione, albumin, vitamin $\mathrm{C}$, bilirubin, uric acid, and others in plasma. ${ }^{39}$ A decrease in TAS was confirmed in an animal model of AMI; after treating these animals with ascorbic acid, TAS significantly increased. ${ }^{40}$ On the other hand, the antioxidant enzyme SOD was elevated in patients with STEMI compared with healthy individuals indicating enhanced antioxidant protection in this acute state, in agreement by the findings of others. ${ }^{41}$ The protective activity of extracellular SOD against oxidative stress and ventricular hypertrophy after myocardial infarct is well known. Furthermore, overexpression of SOD protects the myocardium against ischemia-reperfusion injury. In mice with increased SOD expression, the infarct size was significantly lower. ${ }^{42}$ Superoxide dismutase catalyzes the reaction of the superoxide anion dismutation and is one of the first lines of cell defense against oxidative damage. Since SOD is also localized intracellular, its activity increases due to tissue damage during myocardial infarction, ${ }^{42}$ with a release from the cells as a primary mechanism for increased activity. However, the major argument for discussion remains whether it primarily reflects oxidative stress status or the degree of cell damage in patients with STEMI as a result of free radical burden.

The present study results show that PTX3 correlated negatively while CyPA correlated positively with $\mathrm{SH}$ groups, indicating distinct impact of oxidative stress on both inflammatory parameters in patients with STEMI. The negative correlation between PTX3 and the SH groups as an antioxidant protection parameter possibly indicates that consumption of SH groups in a damage reduction process caused by oxidative stress stimulates the release of PTX3 to enhance the protective effect. According to others, from all examined parameters, total $\mathrm{SH}$ groups have the highest concentration among endogenous antioxidants and probably disturbance in its concentration had the most prominent impact on inflammatory markers. ${ }^{38}$

Enhancement of antioxidant protection is often the result of increased oxidative stress which was also seen in patients with STEMI. The overproduction of ROS accelerates the release of CyPA through activation of transcription factor NF- $\mathrm{kB}$. Yuan et al showed that CyPA-extracellular matrix metalloproteinase inducer interaction in response to ROS is probably key proinflammatory pathway in monocytes. ${ }^{43}$ However, such relationship was not confirmed in the control group indicating a better balance in the oxidative stress parameters of these individuals. Furthermore, HB-EGF positively correlated with PTX3. The relationship between HB-EGF and PTX3 emphasized their joint role in the acute condition studied STEMI group. Our results are in agreement with studies ${ }^{7,8}$ conducted on cell lines and removed tissue where transcription of HB-EGF and PTX3 are dependent of similar activators.

To better understand the effect of different oxidative stress and other risk factors on the occurrence of high concentrations of PTX3 and HB-EGF, we grouped them into several factors according to their common physiological characteristics. "Metabolic-oxidative stress factor" appears as a single most impacting predictor of high PTX3 values. It included glucose and $\mathrm{PAB}$ parameters with positive loadings meaning that PTX3 is high when glucose and oxidative stress measured through $\mathrm{PAB}$ are high. One of the major contributors to the increased ROS generation in the STEMI group is chronic hyperglycemia. In our study, $50 \%$ of patients had a glucose level $>7.0 \mathrm{mmol} / \mathrm{L}$ and only $25 \%$ of them had the values lower than $6.3 \mathrm{mmol} / \mathrm{L}$. Correlation between PAB and glucose is in accordance with findings of Alamdari et $\mathrm{al}^{34}$ and combined effect of those 2 parameters implies that chronic hyperglycemia increases the level of PTX3 and oxidative stress. ${ }^{44}$ An in vitro study reported that hyperglycemia affected monocytes, which led to increased expression of TNF- $\alpha$ through oxidative stress and the NF- $\kappa B$ pathway, ${ }^{45}$ with stimulation of PTX3 secretion. ${ }^{46}$ Since we have found that $\mathrm{CyPA}$ is a predictor of high PTX3 values and considering that CyPA is directly related and correlates with oxidative stress, the question is whether this impact is straightforward. Using multiple regression analysis, the only independent predictors of high PTX3 were glucose and PAB levels. Patients with STEMI have extremely high PTX3 values that most likely will be higher if patients were subjected to higher glucose and oxidative stress levels, which implies why patients with high values of PTX3 have a more unfavorable prognosis. However, this correlation still cannot provide a more accurate prognosis in affected patient population.

The novelty of our work is the analysis of the relationship between examined parameters including a joint effect of oxidative stress and antioxidant predictors on inflammatory parameters. For the first time, in real-world conditions, we 
confirmed that there is a similar activation mechanism for PTX3 and HG-EGF.

This study has some limitations. First, blood was sampled for patients with STEMI after admission to the emergency department, at any time of the day, while from controls, samples were collected according to the current blood sampling protocol, which means after overnight fasting. In this way, the influence of food, daily activities, or circadian rhythm on certain parameters in patients with STEMI could not be avoided. Second, high values were defined in regards to a healthy population but it would be necessary to test if the results would be the same if they were defined relative to stable CVD. Third, sample size was small, and thus the results of this study should be investigated in a large-scale study.

\section{Conclusions}

The observed relationship between HB-EGF and PTX3 acknowledges possible mutual role in acute conditions such as STEMI. Patients with a profile of impaired oxidative stress and antioxidant status evaluated via $\mathrm{PAB}$ and changed glucose metabolism are more likely to have high PTX3 values and a worse prognosis. It appears that PTX3 is the only significant predictor of high HB-EGF values. The findings indicate possibility for clinical use of these biomarkers that should be reassessed in a larger prospective study.

\section{Authors' Note}

VVD performed the analysis, processed the experimental data, and wrote the article. NBS designed and directed the project, performed the statistical analysis, and edited the manuscript. JKS and VSK were involved in planning and supervising of the experiments. MZ and AN aided in interpreting the results and edited the manuscript. LM performed the measurements. MM and AV were involved in planning the project and contributed to sample preparation. SK, II, SH, MC, and MS aided in interpreting the results. All authors approved the final version to be submitted.

\section{Declaration of Conflicting Interests}

The author(s) declared no potential conflicts of interest with respect to the research, authorship, and/or publication of this article.

\section{Funding}

The author(s) disclosed receipt of the following financial support for the research, authorship, and/or publication of this article: This work was funded by a grant from the Ministry of Education, Science and Technological Development, Republic of Serbia (Project No. 175035).

\section{ORCID iD}

Vesna Vuković Dejanović (D) https://orcid.org/0000-0001-6273-8498

\section{References}

1. Börekçi A, Gür M, Türkoğlu C, et al. Oxidative stress and spontaneous reperfusion of infarct-related artery in patients with STsegment elevation myocardial infarction. Clin Appl Thromb Hemost. 2016;22:171-7.
2. Hori M, Nishida K. Oxidative stress and left ventricular remodeling after myocardial infarction. Cardiovasc Res. 2009;81:457-64.

3. Biswas SK. Does the interdependence between oxidative stress and inflammation explain the antioxidant paradox? Oxidat Med Cell Long. 2016;2016:5698931.

4. Satoh K, Nigro P, Berk BC. Oxidative stress and vascular smooth muscle cell growth: a mechanistic linkage by cyclophilin A. Antiox Redox Sign. 2010;12:675-82.

5. Seizer P, Ochmann C, Schönberger T, et al. Disrupting the EMMPRIN (CD147)-cyclophilin A interaction reduces infarct size and preserves systolic function after myocardial ischemia and reperfusion. Arterioscler Thromb Vasc Biol. 2011;31:1377-86.

6. Pinho-Ribeiro F, Hohmann M, Borghi S, et al. Protective effects of the flavonoid hesperidin methyl chalcone in inflammation and pain in mice: role of TRPV1, oxidative stress, cytokines, and NF кB. Chem Biol Interact. 2015;228:88-99.

7. Miyata K, Yotsumoto F, Ouk Nam S, et al. Contribution of a transcription factor, SP1, to the promotion of HB-EGF expression in defense mechanism against the treatment of irinotecan in ovarian clear cell carcinoma. Canc Med. 2014;3:1159-69.

8. Rolph MS, Zimmer S, Bottazzi B, et al. Production of the long pentraxin PTX3 in advanced atherosclerotic plaques. Arterioscler Thromb Vasc Biol. 2002;22:e10-e14.

9. Rattik S, Wigren M, Björkbacka H, et al. High plasma levels of heparin-binding epidermal growth factor are associated with a more stable plaque phenotype and reduced incidence of coronary events. Arterioscler Thromb Vasc Biol. 2015;35:222-28.

10. Makki N, Thiel K, Miller F. The epidermal growth factor receptor and its ligands in cardiovascular disease. Int J Mol Sci. 2013;14: 20597-613.

11. Maugeri N, Rovere-Querini P, Slavich M, et al. Early and transient release of leukocyte pentraxin 3 during acute myocardial infarction. J Immunol. 2011;187:970-9.

12. Kimura S, Inagaki H, Haraguchi G, et al. Relationships of elevated systemic pentraxin-3 levels with high-risk coronary plaque components and impaired myocardial perfusion after percutaneous coronary intervention in patients with ST-elevation acute myocardial infarction. Circ J. 2013; CJ-13.

13. Akgul O, Baycan OF, Bulut U, et al. Long-term prognostic value of elevated pentraxin 3 in patients undergoing primary angioplasty for ST-elevation myocardial infarction. Cor Art Dis. 2015;26:592-7.

14. Ushikoshi H, Takahashi T, Chen X, et al. Local overexpression of HB-EGF exacerbates remodeling following myocardial infarction by activating noncaridiomyocytes. Lab Invest. 2005; 85:862-73.

15. Hervé A, Williams LJ. Principal Component Analysis.Wiley Interdisciplinary Reviews: Computational Statistics 2.4; 2010: 433-59.

16. World Medical Association. World medical association declaration of Helsinki: ethical principles for medical research involving human subjects. JAMA. 2013;310:2191-4.

17. Mach F, Baigent C, Catapano AL, et al. 2019 ESC/EAS Guidelines for the management of dyslipidaemias: lipid modification to reduce cardiovascular risk. Eur Heart J. 2020;41:111-88. 
18. Witko-Sarsat V, Friedlander M, Capeillere-Blandin C, et al. Advanced oxidation protein products as a novel marker of oxidative stress in uremia. Kidney Int. 1996;49:1304-13.

19. Ellman GL. Tissue sulphydryl groups. Arch Biochem Biophys. 1959;82:70-7.

20. Erel O. A new automated colorimetric method for measuring total oxidant status. Clin Biochem. 2005;38:1103-11.

21. Alamdari DH, Paletas K, Pegiou T, et al. Novel assay for the evaluation of prooxidant-antioxidant balance, before and after antioxidant vitamin administration in type II diabetes patients. Clin Biochem. 2007;40:248-54.

22. Erel O. A novel automated method to measure total antioxidant response against potent free radical reactions. Clin Biochem. 2004;37:112-9.

23. Misra HP, Fridovich I. The role of superoxide anion in the autooxidation of epinephrine and a simple assay for superoxide dismutase. J Biol Chem. 1972;247:3170-5.

24. Ludbrook J. Advantages of permutation (randomization) tests in clinical and experimental pharmacology and physiology. Clin Exp Pharmacol Physiol. 1994;21:673-86.

25. Youden WJ. Index for rating diagnostic tests. Cancer. 1950;3: 32-5.

26. Tomandlova M, Jarkovsky J, Tomandl J, et al. Prognostic value of pentraxin-3 level in patients with STEMI and its relationship with heart failure and markers of oxidative stress. Disease Markers. 2015;2015:159051.

27. Peri G, Introna M, Corradi D, et al. PTX3, a prototypical long pentraxin, is an early indicator of acute myocardial infarction in humans. Circ. 2000;102:636-41.

28. Helseth R, Solheim S, Opstad T, et al. The time profile of pentraxin 3 in patients with acute ST-elevation myocardial infarction and stable angina pectoris undergoing percutaneous coronary intervention. Med Inflam. 2014;2014:608414.

29. Fornai F, Carrizzo A, Forte M, et al. The inflammatory protein pentraxin 3 in cardiovascular disease. Immun Ageing. 2016;13:25.

30. Tanaka N, Masamura K, Yoshida M, et al. A role of heparinbinding epidermal growth factor-like growth factor in cardiac remodeling after myocardial infarction. Biochem Biophys Res Comm. 2002;297:375-81.

31. Nigro P, Pompilio G, Capogrossi MC. Cyclophilin A: a key player for human disease. Cell Death Dis. 2013;4:e888.

32. Nikolic-Heitzler V, Rabuzin F, Tatzber F, et al. Persistent oxidative stress after myocardial infarction treated by percutaneous coronary intervention. Tohoku J Exp Med. 2006; 210:247-55.

33. Lo Presti R, Catania A, D'Amico T, et al. Oxidative stress in young subjects with acute myocardial infarction: Evaluation at the initial stage and after 12 months. Clin Appl Thromb Hemost. 2008; $14: 421-7$.

34. Alamdari D, Ghayour-Mobarhan M, Tavallaie S, et al. Prooxidant-antioxidant balance as a new risk factor in patients with angiographically defined coronary artery disease. Clin Biochem. 2008;41:375-80.

35. Parizadeh S, Azarpazhooh M, Moohebati M, et al. Simvastatin therapy reduces prooxidant-antioxidant balance: results of a placebo-controlled cross-over trial. Lipids. 2011;46:333-40.

36. Sitar ME, Aydin S, Çataky U. Human serum albumin and its relation with oxidative stress. Clin Lab. 2013;59:945-52.

37. Kotur-Stevuljevic J, Bogavac-Stanojevic N, Jelic-Ivanovic Z, et al. Oxidative stress and paraoxonase 1 status in acute ischemic stroke patients. Atherosclerosis. 2015;241:192-8.

38. Tanriverdi F, Kavakh H, Celik G, et al. Evaluation of serum thiol levels in patients with the non-ST-elevation acute coronary syndrome. J Exp Clin Med. 2013;30:295-9.

39. Guerin P, Bigot E, Patrice T. Evidence for antioxidants consumption in the coronary blood of patients with acute myocardial infarction. J Thromb Thrombolysis. 2013;35:41-7.

40. Yao D, Vlessidis AG, Evmiridis NP, et al. Possible mechanism for nitric oxide and oxidative stress-induced pathophysiological variance in acute myocardial infarction development. a study by a flow injection-chemiluminescence method. Anal Chim Acta. 2004;505:115-23.

41. Lavall MC, Bonfanti G, Ceolin RB, et al. Oxidative profile of patients with ST-segment elevation myocardial infarction. Clin Lab. 2016;62:971-3.

42. van Deel ED, Lu Z, Xu X, et al. Extracellular SOD protects the heart against oxidative stress and hypertrophy after myocardial infarction. Free Radic Biol Med. 2008;44:1305-13.

43. Yuan W, Ge H, He B. Pro-inflammatory activities induced by CyPA-EMMPRIN interaction in monocytes. Atherosclerosis. 2010;213:415-21.

44. Takashi Y, Koga M, Matsuzawa Y, et al. Circulating pentraxin 3 is positively associated with chronic hyperglycemia but negatively associated with plasma aldosterone concentration. PloS one. 2018;13:e0196526.

45. Shanmugam N, Reddy MA, Guha M, Natarajan R. High glucoseinduced expression of proinflammatory cytokine and chemokine genes in monocytic cells. Diabetes. 2003;52:1256-64.

46. Bastrup-Birk S, Skjoedt MO, Munthe-Fog L, et al. Pentraxin-3 serum levels are associated with disease severity and mortality in patients with systemic inflammatory response syndrome. PLoS One. 2013;8:e73119. 